\title{
Zero Padding or Cyclic Prefix: Evaluation for Non-Orthogonal Signals
}

\author{
Waseem Ozan, Student Member, IEEE, Ryan Grammenos, Member, IEEE, Izzat Darwazeh, Senior Member, IEEE
}

\begin{abstract}
The debate of using zero padding (ZP) instead of a cyclic prefix (CP) for enhancing channel estimation and equalization performance is a recurring topic. This is particularly true for orthogonal signals, such as orthogonal frequency division multiplexing (OFDM). Yet, there are far fewer studies evaluating the impact of $\mathrm{ZP}$ and $\mathrm{CP}$ in non-orthogonal systems. Such systems have the added complexity of self-induced interference rendering channel estimation and equalization more challenging. For this reason, this work proposes a new channel estimation and equalization technique for non-orthogonal systems, which combines ZP with an orthogonal demodulator. Results show that the multipath components that appear in the ZP part can be used to enhance performance when compared to the CP approach.
\end{abstract}

Index Terms-zero padding, cyclic prefix, non-orthogonal, FDM, NB-IoT.

\section{INTRODUCTION}

In a classic paper published in 2002 [1], it was demonstrated that using zero padding (ZP) instead of a conventional nonzero cyclic prefix (CP) for orthogonal signals, such as orthogonal frequency division multiplexing (OFDM), can improve channel estimation and guarantee symbol recovery. This debate of using ZP or CP was revisited in 2016 for OFDM [2], yet this time considering the use of filtering or windowing to improve spectral performance required in fifth generation $(5 \mathrm{G})$ communication systems.

This widely accepted paradigm of using orthogonal signals, however, has been challenged in recent times by considering the use of non-orthogonal signals to enhance efficiency and scalability [3]. A number of candidate waveform techniques are being considered for beyond 5G communications [4] and [5], where these new waveform techniques can be classified into two groups according to their aim. The first group aims to reduce the out-of-band (OOB) spectral leakage, which is achieved by several methods, such as generalized frequency division multiplexing (GFDM) where each subcarrier is filtered and Filterbank Based Multicarrier (FBMC) where each subcarrier is pulse shaped. The second group aims to increase transmission rate by reducing the transmission time, such as faster-than-Nyquist (FTN) or enhancing spectral effieciency by saving signal bandwidth, such as spectrally efficient frequency division multiplexing (SEFDM). In this work we use SEFDM as an example.

SEFDM is a non-orthogonal multi-carrier scheme, which packs more subcarriers into the same spectrum relative to OFDM, thereby improving spectral efficiency [6] and making it a topic of current interest [7], [8]. Nevertheless, the self-

The authors acknowledge the sponsorship of W. Ozan's PhD by UCL EE department.

The authors are with the Department of Electronic and Electrical Engineering, University College London (UCL), London WC1E 7JE, U.K. (e-mail: \{w.ozan; r.grammenos; i.darwazeh\}@ucl.ac.uk). created inter-carrier interference (ICI) inherent in SEFDM systems combined with multipath effects renders channel estimation and equalization more challenging compared to OFDM [9], [10].

In [10], channel estimation were carried out in the timedomain. It has been shown that time-domain channel estimation techniques provide a good estimate, and subsequently, a good equalization of the channel [10]. However, the computational complexity is relatively high since time-domain estimation requires at least one matrix inversion operation to perform the de-convolution process needed to estimate the channel. The work in [10], reports that the mean-square error (MSE) reaches an error floor at high $E_{b} / N_{o}$ values, when channels are estimated using CP-SEFDM pilots. The work in optical SEFDM [11] uses OFDM pilot symbols for channel estimation, however, an interpolation process is needed to compute the channel estimate for SEFDM subcarriers. More recently, a new channel estimation method utilises OFDM pilot symbols [9], which are longer in time-domain in comparison to SEFDM symbols. Hence, this requires modification in standard resource blocks, such as long-term evolution (LTE) resource blocks [12].

To address this challenge, this letter presents the following novel contributions; first, we argue and show that using the proposed ZP scheme in SEFDM results in channel estimation and equalization expressions free of interference terms, as depicted in equations (29) and (30). On the contrary, using CP in SEFDM results in interference terms for channel estimation and equalization, which are depicted in equations (20) and (21); second, we show how an orthogonal demodulator in conjunction with a ZP scheme can improve channel estimation and equalization in non-orthogonal systems; third, our numerical results demonstrate that ZP-SEFDM outperforms CP-SEFDM, the latter reaching a non-zero error floor, as shown in the performance results section.

\section{SEFDM SIGNAL MODEL}

SEFDM signals consist of a stream of multi-carrier symbols, where each multi-carrier symbol in turn carries $N$ complex data symbols $\mathbf{d}=\left[d_{1}, \ldots, d_{N}\right]^{T} \in \mathbb{C}^{N \times 1}$. The complex data symbols are oversampled (padded with zeros) before the modulation process. The oversampling rate is given by $\rho=Q / N$, where $Q$ is the number of time samples in a single SEFDM symbol. This means that only $N$ out of the $Q$ subcarriers are active. The input data symbols that are fed into the modulation process are given as:

$$
s_{n}= \begin{cases}d_{n}, & 0 \leq n<N \\ 0, & N \leq n<Q-1\end{cases}
$$


Each data symbol, $s_{n}$, is modulated onto one subcarrier. The discrete-time SEFDM symbol is expressed as:

$$
x(k)=\frac{1}{\sqrt{Q}} \sum_{n=0}^{Q-1} s_{n} e^{\left(\frac{j 2 \pi \alpha n k}{Q}\right)}, k=0, \ldots, Q-1
$$

where $k \in \mathbb{Z}$ is the time sample index, $n \in \mathbb{Z}$ is the frequency domain subcarrier index and $\alpha \leq 1$ is the bandwidth compression factor defined in SEFDM systems [6].

The discrete SEFDM symbol is given in matrix form as:

$$
\mathbf{x}=\mathbf{F s}
$$

where $\mathbf{x} \in \mathbb{C}^{Q \times 1}$ is a vector of time samples representing one SEFDM symbol; $\mathbf{F} \in \mathbb{C} Q \times Q$ is the sampled non-orthogonal subcarrier matrix used in the SEFDM modulation process [10] and $\mathbf{s} \in \mathbb{C}^{Q \times 1}$ is the vector of input data symbols. The matrix elements of $\mathbf{F}$ are given by $\mathbf{F}_{k, n}=\frac{1}{\sqrt{Q}} e^{(j 2 \pi \alpha n k / Q)}$.

\section{SEFDM WITH CYCLIC PREFIX}

Using the conventional CP technique in SEFDM systems [9], the last $\mu \in \mathbb{N}$ samples of an SEFDM symbol, $\overline{\mathbf{x}}_{c p} \in \mathbb{C}^{\mu \times 1}$, are added to the beginning of each transmitted SEFDM symbol giving:

$$
\mathbf{x}_{c p}=\left[\begin{array}{c}
\overline{\mathbf{x}}_{c p} \\
\mathbf{x}
\end{array}\right]
$$

where $\mathbf{x}_{c p} \in \mathbb{C}^{(Q+\mu) \times 1}$ is the CP-SEFDM symbol. The CPSEFDM symbol is transmitted through a wireless frequency selective channel with channel impulse response $(\mathrm{CIR}) \mathbf{h}=$ $\left[h_{0}, h_{1}, \ldots, h_{\mu}\right] \in \mathbb{C}^{(\mu+1) \times 1}$. The mathematical representation of the received CP-SEFDM after traversing a multipath channel, $\mathbf{y}_{c p} \in \mathbb{C}^{(Q+2 \mu) \times 1}$, is given in a linear convolution process of CP-SEFDM symbol with the CIR as:

$$
\mathbf{y}_{c p}=\left[\begin{array}{c}
\overline{\mathbf{y}}_{c p} \\
\mathbf{y} \\
\mathbf{y}_{i s i}
\end{array}\right]=\mathbf{h} * \mathbf{x}_{c p}
$$

where $\overline{\mathbf{y}}_{c p} \in \mathbb{C}^{\mu \times 1}$ is the $\mathrm{CP}$ part that is affected by the inter-symbol interference (ISI) components stemming from the previous SEFDM symbol and hence it is ignored at the receiver; $\mathbf{y} \in \mathbb{C}^{Q \times 1}$ is the received SEFDM symbol that is passed to the detection stage; $\mathbf{y}_{i s i} \in \mathbb{C}^{\mu \times 1}$ represents the undesired ISI components that are added to the succeeding SEFDM symbol and [*] denotes a linear convolution process.

At the receiver, the symbols will arrive distorted by the channel and contaminated with additive white Gaussian noise (AWGN). The use of a CP at the beginning of a symbol results in the channel impulse response being modelled as a circulant convolution matrix, $\mathbf{H}_{c p} \in \mathbb{C}^{Q \times Q}$ [13]. Thus, the received SEFDM symbol, $\mathbf{y}$, is represented as:

$$
\mathbf{y}=\mathbf{H}_{c p} \mathbf{x}+\mathbf{z}=\mathbf{H}_{c p} \mathbf{F s}+\mathbf{z}
$$

where $\mathbf{z} \in \mathbb{C}^{Q \times 1}$ is the AWGN noise vector.

The eigenvalue decomposition of the channel matrix, $\mathbf{H}_{c p}$, is given as [13], [14]:

$$
\mathbf{H}_{c p}=\mathbf{M}_{c p} \boldsymbol{\Lambda}_{c p} \mathbf{M}_{c p}^{H}
$$

where $\boldsymbol{\Lambda}_{c p}=\operatorname{diag}\left\{\lambda_{0}, \ldots, \lambda_{Q-1}\right\} \in \mathbb{C}^{Q \times Q}$ and $\lambda_{i}$ is the $i$ th eigenvalue of $\mathbf{H}_{c p}$, while $\mathbf{M}_{c p}$ and $\mathbf{M}_{c p}^{H} \in \mathbb{C} Q \times Q$ are unitary matrices, where $\mathbf{M}_{c p}^{H}$ has rows that are the eigenvectors of $\mathbf{H}_{c p}$ and [.] ${ }^{H}$ denotes the conjugate transpose operation. Every eigenvector, $\mathbf{e}_{v} \in \mathbb{C}^{1 \times Q}$, of $\mathbf{H}_{c p}$ is given as [14]:

$$
e_{v}(p)=\frac{1}{\sqrt{Q}}\left\{W_{Q}^{0 p}, W_{Q}^{1 p}, \ldots, W_{Q}^{(Q-1) p}\right\}, p=0, \ldots, Q-1
$$

where $p \in \mathbb{Z}$ is the row index of $\mathbf{H}_{c p}$ and $W_{Q}=e^{-j 2 \pi / Q} \in \mathbb{C}$. From (8), it can be shown [14] that $\mathbf{M}_{c p}^{H}$ is a discrete Fourier transform (DFT) matrix.

\section{A. Subcarrier matrix based receiver}

The demodulated SEFDM signal, $\mathbf{r}_{c p}$, using the transpose conjugate of the non-orthogonal carrier matrix, $\mathbf{F}^{H}$, is expressed in matrix form as:

$$
\begin{aligned}
\mathbf{r}_{c p} & =\mathbf{F}^{H} \mathbf{y}=\mathbf{F}^{H}\left[\mathbf{H}_{c p} \mathbf{x}+\mathbf{z}\right] \\
& =\mathbf{F}^{H}\left[\mathbf{H}_{c p} \mathbf{F} \mathbf{s}+\mathbf{z}\right]=\mathbf{F}^{H}\left[\mathbf{M}_{c p} \boldsymbol{\Lambda}_{c p} \mathbf{M}_{c p}^{H} \mathbf{F s}+\mathbf{z}\right] \\
& =\mathbf{F}^{H} \mathbf{M}_{c p} \boldsymbol{\Lambda}_{c p} \mathbf{M}_{c p}^{H} \mathbf{F s}+\mathbf{F}^{H} \mathbf{z}=\mathbf{A} \mathbf{\Lambda}_{c p} \mathbf{B s}+\mathbf{F}^{H} \mathbf{z}
\end{aligned}
$$

where the components of the $m^{\text {th }}$ row and $n^{\text {th }}$ column of the matrices $\mathbf{A}=\mathbf{F}^{H} \mathbf{M}_{c p}$ and $\mathbf{B}=\mathbf{M}_{c p}^{H} \mathbf{F}$ can be found as:

$$
\mathbf{A}[m, n]=\frac{1}{Q} \sum_{k=0}^{Q-1} e^{\left(\frac{-j 2 \pi \alpha m k}{Q}\right)} e^{\left(\frac{j 2 \pi n k}{Q}\right)}=\frac{1}{Q}\left[\frac{1-e^{-j 2 \pi(\alpha m-n)}}{1-e^{\frac{-j 2 \pi(\alpha m-n)}{Q}}}\right]
$$

where $k, m \& n=0, \ldots, Q-1 \in \mathbb{Z}$. The derivation of (10) is based on a sum of geometric series: $\sum_{k=0}^{Q-1} r^{k}=\frac{1-r}{1-r}$. Following the same method applied in (10), the components of matrix $\mathbf{B}$ are found as:

$$
\mathbf{B}[m, n]=\frac{1}{Q} \sum_{k=0}^{Q-1} e^{\left(\frac{-j 2 \pi m k}{Q}\right)} e^{\left(\frac{j 2 \pi \alpha n k}{Q}\right)}=\frac{1}{Q}\left[\frac{1-e^{-j 2 \pi(m-\alpha n)}}{1-e^{\frac{-j 2 \pi(m-\alpha n)}{Q}}}\right]
$$

When $\alpha=1$, the signals are OFDM and hence $\mathbf{A}$ and $\mathbf{B}$ are identity matrices so (9) becomes:

$$
\mathbf{r}_{c p}=\Lambda_{c p} \mathbf{s}+\mathbf{F}^{H} \mathbf{z}
$$

which shows that the multipath channel is decomposed into a set of $Q$ orthogonal subchannels. On the contrary, we recall that in SEFDM, $\alpha<1$, which implies that $\mathbf{A}$ and $\mathbf{B}$ are no longer identity matrices. Thus, the wideband channel cannot be decomposed into independent narrowband subchannels.

To overcome this limitation, this work proposes for the first time in SEFDM systems, the use of an orthogonal DFTbased demodulator at the receiver to recover the data symbols which were modulated using a non-orthogonal modulator at the transmitter.

\section{B. DFT based receiver}

Each received SEFDM symbol is appended with zeros to length $V=Q / \alpha$ before the symbol is fed to a $V$-point DFT. Assuming that $Q / \alpha \in \mathbb{N}$, then (6) may be re-written as:

$$
\begin{aligned}
{\left[\begin{array}{c}
\mathbf{y} \\
\mathbf{0}_{(V-Q) \times 1}
\end{array}\right] } & =\left[\begin{array}{cc}
\mathbf{H}_{c p}, & \mathbf{0}_{Q \times(V-Q)} \\
\mathbf{0}_{(V-Q) \times Q}, & \mathbf{0}_{(V-Q) \times(V-Q)}
\end{array}\right]\left[\begin{array}{c}
\mathbf{F} \\
\mathbf{0}_{(V-Q) \times Q}
\end{array}\right] \mathbf{s} \\
& +\left[\begin{array}{c}
\mathbf{z} \\
\mathbf{0}_{(V-Q) \times 1}
\end{array}\right] \\
\tilde{\mathbf{y}} & =\tilde{\mathbf{H}}_{c p} \tilde{\mathbf{F}} \mathbf{s}+\tilde{\mathbf{z}}=\tilde{\mathbf{H}}_{c p} \tilde{\mathbf{x}}+\tilde{\mathbf{z}}
\end{aligned}
$$


where $\tilde{\mathbf{y}} \in \mathbb{C}^{V \times 1}, \tilde{\mathbf{H}}_{c p} \in \mathbb{C}^{V \times V}, \tilde{\mathbf{F}} \in \mathbb{C}^{V \times Q}, \tilde{\mathbf{x}} \in \mathbb{C}^{V \times 1}$ and $\tilde{\mathbf{z}} \in \mathbb{C}^{V \times 1}$ are the zero appended versions of the received SEFDM symbol, channel matrix, subcarrier matrix, transmitted SEFDM symbol and AWGN noise vector, respectively.

The appended channel matrix, $\tilde{\mathbf{H}}_{c p}$, is no longer circulant and thus cannot be decomposed. This matrix is expressed as:

$$
\tilde{\mathbf{H}}_{c p}=\left[\begin{array}{cccccccccc}
h_{0} & 0 & \ldots & 0 & h_{\mu} & \ldots & h_{1} & 0 & \ldots & 0 \\
h_{1} & h_{0} & \ddots & & \ddots & \ddots & \vdots & \vdots & & \vdots \\
\vdots & h_{1} & \ddots & \ddots & & \ddots & h_{\mu} & \vdots & & \vdots \\
h_{\mu} & \vdots & \ddots & h_{0} & \ddots & & \ddots & \vdots & & \vdots \\
0 & h_{\mu} & \ddots & h_{1} & \ddots & \ddots & & 0 & & \vdots \\
\vdots & \ddots & \ddots & \vdots & \ddots & \ddots & \ddots & & & \vdots \\
\vdots & & \ddots & h_{\mu} & \ldots & h_{1} & h_{0} & 0 & & \vdots \\
\vdots & & & 0 & \ldots & \ldots & \ldots & 0 & & \vdots \\
\vdots & & & & & & & & & \vdots \\
0 & \ldots & \ldots & \ldots & \ldots & 0 & \ldots & \ldots & \ldots & 0
\end{array}\right]
$$

Following a similar approach to that outlined in [15], $\tilde{\mathbf{H}}_{c p}$ can be constructed from three $V \times V$ matrices as:

$$
\tilde{\mathbf{H}}_{c p}=\mathbf{H}_{c p 1}+\mathbf{H}_{c p 2}-\mathbf{H}_{c p 3}
$$

where $\mathbf{H}_{c p 1} \in \mathbb{C}^{V \times V}$ is the desired circulant matrix having tap coefficient vectors $\mathbf{h}^{T}=\left[h_{0}, \ldots, h_{\mu}\right]$, while $\mathbf{H}_{c p 2} \in \mathbb{C}^{V \times V}$ and $\mathbf{H}_{c p 3} \in \mathbb{C}^{V \times V}$ represent the missing and unwanted elements in the appended channel matrix, $\tilde{\mathbf{H}}_{c p}$. The elements of $\mathbf{H}_{c p 2}$ and $\mathbf{H}_{c p 3}$ are given as:

$$
\begin{aligned}
\mathbf{H}_{c p 2} & =\left[\begin{array}{ccccccccc}
0 & \ldots & 0 & h_{\mu} & \ldots & h_{1} & 0 & \ldots & 0 \\
\vdots & & & \ddots & \ddots & \vdots & \vdots & & \vdots \\
\vdots & & & & \ddots & h_{\mu} & \vdots & & 0 \\
\vdots & & & & & \ddots & 0 & & 0 \\
\vdots & & & & & & \ddots & \ddots & \vdots \\
0 & \ldots & \ldots & \ldots & \ldots & \ldots & \ldots & 0 & 0
\end{array}\right] \\
\mathbf{H}_{c p 3} & =\left[\begin{array}{cccccccccc}
0 & \ldots & \ldots & \ldots & \ldots & 0 & h_{\mu} & \ldots & h_{1} \\
\vdots & & & & & & \ddots & \ddots & \vdots \\
0 & & & & & & & \ddots & h_{\mu} \\
\vdots & \ddots & 0 & \ldots & \ldots & 0 & \ldots & \ldots & 0 \\
\vdots & & \ddots & h_{\mu} & \ldots & h_{1} & h_{0} & \ddots & \vdots \\
\vdots & & & \ddots & h_{\mu} & \vdots & h_{1} & h_{0} & 0 \\
0 & \ldots & \ldots & \ldots & 0 & h_{\mu} & \vdots & h_{1} & h_{0}
\end{array}\right]
\end{aligned}
$$

The eigenvalue decomposition of $\mathbf{H}_{c p 1}$ is given as:

$$
\mathbf{H}_{c p 1}=\mathbf{M} \mathbf{\Lambda} \mathbf{M}^{H}
$$

Applying the concept from (8), $\boldsymbol{\Phi}^{H}=\mathbf{M}^{H}$, where $\boldsymbol{\Phi}^{H}$ is a DFT matrix of size $V \times V$ [14]. The demodulated CP-SEFDM signal, $\tilde{\mathbf{r}}_{c p}$, is thereby expressed in matrix form as:

$$
\begin{aligned}
\tilde{\mathbf{r}}_{c p} & =\boldsymbol{\Phi}^{H} \tilde{\mathbf{y}}=\boldsymbol{\Phi}^{H}\left[\mathbf{H}_{c p 1} \tilde{\mathbf{x}}+\mathbf{H}_{c p 2} \tilde{\mathbf{x}}-\mathbf{H}_{c p 3} \tilde{\mathbf{x}}+\tilde{\mathbf{z}}\right] \\
& =\boldsymbol{\Phi}^{H}\left[\mathbf{M} \mathbf{\Lambda} \mathbf{M}^{H} \tilde{\mathbf{x}}+\mathbf{H}_{c p 2} \tilde{\mathbf{x}}-\mathbf{H}_{c p 3} \tilde{\mathbf{x}}+\tilde{\mathbf{z}}\right] \\
& =\mathbf{M}^{H} \mathbf{M} \mathbf{\Lambda} \mathbf{M}^{H} \tilde{\mathbf{F}} \mathbf{s}+\boldsymbol{\Phi}^{H} \mathbf{H}_{c p 2} \tilde{\mathbf{x}}-\boldsymbol{\Phi}^{H} \mathbf{H}_{c p 3} \tilde{\mathbf{x}}+\boldsymbol{\Phi}^{H} \tilde{\mathbf{z}} \\
& =\boldsymbol{\Lambda} \boldsymbol{\Gamma} \mathbf{s}+\boldsymbol{\Phi}^{H} \mathbf{H}_{c p 2} \tilde{\mathbf{x}}-\boldsymbol{\Phi}^{H} \mathbf{H}_{c p 3} \tilde{\mathbf{x}}+\boldsymbol{\Phi}^{H} \tilde{\mathbf{z}} \\
& =\boldsymbol{\lambda} \odot \tilde{\mathbf{s}}+\tilde{\mathbf{r}}_{c p 2}-\tilde{\mathbf{r}}_{c p 3}+\tilde{\mathbf{z}}_{c p}
\end{aligned}
$$

where $\boldsymbol{\Lambda}$ is the diagonal matrix of the narrowband subchannel gains, whose diagonal elements are the subchannel gain vector $\lambda \in \mathbb{C}^{V \times 1}, \tilde{\mathbf{s}}=\boldsymbol{\Gamma} \mathbf{s}$ is expected received SEFDM symbol when no multipath or noise channels are present, $\mathbf{M}^{H} \mathbf{M}$ is equal to an identity matrix, $\boldsymbol{\Gamma}=\mathbf{M}^{H} \tilde{\mathbf{F}} \in \mathbb{C}^{V \times Q}$ is the SEFDM correlation matrix, which quantifies the interference contribution to each subcarrier from its neighbouring subcarriers [10], and the notation $(\odot)$ is the element-wise multiplication. It should be evident that even in the absence of noise, the demodulated CP-SEFDM signal would comprise interference components from the missing and unwanted matrices, $\mathbf{H}_{c p 2}$ and $\mathbf{H}_{c p 3}$.

\section{Channel Estimation and Equalization in CP-SEFDM}

The analytical expression of channel estimation for CPSEFDM is found using zero forcing (ZF) and it is given by:

$$
\begin{aligned}
\hat{\boldsymbol{\lambda}} & =\tilde{\mathbf{r}}_{c p} \cdot / \tilde{\mathbf{s}}=\left(\boldsymbol{\lambda} \odot \tilde{\mathbf{s}}+\tilde{\mathbf{r}}_{c p 2}-\tilde{\mathbf{r}}_{c p 3}+\tilde{\mathbf{z}}_{c p}\right) \cdot / \tilde{\mathbf{s}} \\
& =\underbrace{\lambda}_{1}+\underbrace{\boldsymbol{\lambda}}_{2} \underbrace{\tilde{\mathbf{r}}_{c p 3} \cdot / \tilde{\mathbf{s}}}_{3 p 2 \cdot / \tilde{\mathbf{s}}}+\underbrace{\tilde{\tilde{\mathbf{z}}_{c p} \cdot / \tilde{\mathbf{s}}}}_{3}
\end{aligned}
$$

where part 1 represents the subchannel gain estimated at each subcarrier, parts 2 and 3 are the added interference, part 4 corresponds to the noise signal, and the division operator notation (./) between the two vectors in equations (20), (21), (29) and (30), is the element-wise division.

The analytical expression of channel equalization for CPSEFDM is found using one-tap equalizer and it is given as:

$$
\begin{aligned}
\hat{\mathbf{r}}_{c p} & =\tilde{\mathbf{r}}_{c p} \cdot / \boldsymbol{\lambda}=\left(\boldsymbol{\lambda} \odot \tilde{\mathbf{s}}+\tilde{\mathbf{r}}_{c p 2}-\tilde{\mathbf{r}}_{c p 3}+\tilde{\mathbf{z}}_{c p}\right) \cdot / \boldsymbol{\lambda} \\
& =\underbrace{\tilde{\mathbf{s}}}_{1}+\underbrace{\tilde{\mathbf{r}}_{c p 2} \cdot / \boldsymbol{\lambda}}_{2}-\underbrace{\tilde{\mathbf{r}}_{c p 3} \cdot / \boldsymbol{\lambda}}_{3}+\underbrace{\tilde{\mathbf{z}}_{c p} \cdot / \boldsymbol{\lambda}}_{4}
\end{aligned}
$$

where part 1 is the desired signal, which in turn is contaminated with interference (parts 2 and 3) and noise (part 4). The reason of using $\boldsymbol{\lambda}$ in (21) instead of $\hat{\boldsymbol{\lambda}}$ is to depict the analytical expression of channel equalization without considering channel estimation impairments.

\section{SEFDM WITH ZERO PADDING}

Here, each transmitted SEFDM symbol, $\mathbf{x}$, is padded with zeros instead of appending a $\mathrm{CP}$ at the beginning of the SEFDM symbol. The transmitted SEFDM symbol may then be represented as:

$$
\mathbf{x}_{z p}=\left[\begin{array}{c}
\mathbf{x} \\
\mathbf{0}_{\mu \times 1}
\end{array}\right]
$$

The ZP-SEFDM symbol after traversing a multipath wireless channel, $\mathbf{y}_{\text {sym }}$, is given by:

$$
\mathbf{y}_{s y m}=\left[\begin{array}{c}
\tilde{\mathbf{y}} \\
\overline{\mathbf{y}}_{z p} \\
\mathbf{0}_{\mu \times 1}
\end{array}\right]=\mathbf{h} * \mathbf{x}_{z p}
$$


where $\tilde{\mathbf{y}} \in \mathbb{C}^{Q \times 1}$ is the SEFDM symbol affected by the multipath and AWGN channels, $\overline{\mathbf{y}}_{z p} \in \mathbb{C}^{\mu \times 1}$ is the ZP part that contains the energy spillage arising from ISI, and the $\mathbf{0}_{\mu \times 1}$ represents the zeros that result from convolving the CIR and the zeros in the $\mathrm{ZP}$ part, and which are added to the succeeding symbol. Thus, at the receiver, the received ZPSEFDM symbol, $\tilde{\mathbf{y}}$, and its ZP part, $\overline{\mathbf{y}}_{z p}$, can be represented as:

$$
\mathbf{y}_{z p}=\left[\begin{array}{c}
\tilde{\mathbf{y}} \\
\overline{\mathbf{y}}_{z p}
\end{array}\right]=\mathbf{h} * \mathbf{x}+\mathbf{z}_{z p}
$$

where $\mathbf{y}_{z p} \in \mathbb{C}^{(Q+\mu) \times 1}$ is the received symbol with the ZP part, $\mathbf{h} \in \mathbb{C}^{\mu \times 1}$ is the CIR, $\mathbf{x} \in \mathbb{C}^{Q \times 1}$ is the transmitted SEFDM symbol and $\mathbf{z}_{z p} \in \mathbb{C}^{(Q+\mu) \times 1}$ is the AWGN noise vector.

To determine the circulant channel matrix in ZP-SEFDM, the received SEFDM symbol, in which the ZP part carries ISI, is padded with zeros to length $V+\mu$, where $V=Q / \alpha$, and expressed in matrix form as:

$$
\tilde{\mathbf{y}}_{z p}=\left[\begin{array}{c}
\mathbf{y}_{z p} \\
\mathbf{0}_{(V-Q) \times 1}
\end{array}\right]=\left[\begin{array}{c}
\mathbf{h} * \mathbf{x}+\mathbf{z}_{z p} \\
\mathbf{0}_{(V-Q) \times 1}
\end{array}\right]=\mathbf{h} * \tilde{\mathbf{x}}+\tilde{\mathbf{z}}_{z p}
$$

where $\tilde{\mathbf{x}}$ and $\tilde{\mathbf{z}}_{z p}$ are the padded versions of $\mathbf{x}$ and $\mathbf{z}_{z p}$, to length $V$ and $V+\mu$, respectively. Equation (25) can be further analyzed as:

$$
\begin{aligned}
\tilde{\mathbf{y}}_{z p} & =\mathbf{H}_{z p} \tilde{\mathbf{x}}+\mathbf{z}_{z p}=\mathbf{H}_{z p}\left[\begin{array}{c}
\mathbf{x} \\
\mathbf{0}_{(V-Q) \times 1}
\end{array}\right]+\left[\begin{array}{c}
\mathbf{z}_{z p} \\
\mathbf{0}_{(V-Q) \times 1}
\end{array}\right] \\
& =\mathbf{H}_{z p}\left[\begin{array}{c}
\mathbf{F} \\
\mathbf{0}_{(V-Q) \times Q}
\end{array}\right] \mathbf{s}+\tilde{\mathbf{z}}_{z p}=\mathbf{H}_{z p} \tilde{\mathbf{F}} \mathbf{s}+\tilde{\mathbf{z}}_{z p}
\end{aligned}
$$

where $\tilde{\mathbf{y}}_{z p} \in \mathbb{C}^{(V+\mu) \times 1}, \mathbf{H}_{z p} \in \mathbb{C}^{(V+\mu) \times V}, \tilde{\mathbf{F}} \in \mathbb{C}^{V \times Q}, \mathbf{s} \in \mathbb{C}^{Q \times 1}$ and $\tilde{\mathbf{z}}_{z p} \in \mathbb{C}^{(V+\mu) \times 1}$.

In a similar manner to the overlap-add method of block convolution described in [1], we can split the vector $\tilde{\mathbf{y}}_{z p}$ into two parts. The upper part $\mathbf{y}_{u}=\mathbf{H}_{u} \tilde{\mathbf{x}}+\mathbf{z}_{u} \in \mathbb{C}^{V \times 1}$ and the lower part $\mathbf{y}_{l}=\mathbf{H}_{l} \tilde{\mathbf{x}}+\mathbf{z}_{l} \in \mathbb{C}^{\mu \times 1}$, where $\mathbf{H}_{u} \in \mathbb{C}^{V \times V}$ and $\mathbf{H}_{l} \in \mathbb{C}^{\mu \times V}$ correspond to the upper and lower partitions of $\mathbf{H}_{z p}$, respectively. We append $\mathbf{y}_{l}$ with $V-\mu$ zeros to be the same size as $\mathbf{y}_{u}$ and then we add the result to $\mathbf{y}_{u}$. Hence, (26) can be formed as:

$$
\begin{aligned}
\mathbf{y}_{V} & =\mathbf{y}_{u}+\left[\begin{array}{c}
\mathbf{y}_{l} \\
\mathbf{0}_{(V-\mu) \times 1}
\end{array}\right] \\
& =\left(\mathbf{H}_{u}+\left[\begin{array}{c}
\mathbf{H}_{l} \\
\mathbf{0}_{(V-\mu) \times V}
\end{array}\right]\right) \tilde{\mathbf{x}}+\mathbf{z}_{v}=\tilde{\mathbf{H}}_{z p} \tilde{\mathbf{x}}+\mathbf{z}_{v}
\end{aligned}
$$

where $\tilde{\mathbf{H}}_{z p}$ is a $V \times V$ circulant matrix and hence its eigenvalue decomposition is $\tilde{\mathbf{H}}_{z p}=\mathbf{M} \boldsymbol{\Lambda}_{z p} \mathbf{M}^{H}$ with $\boldsymbol{\Phi}^{H}=\mathbf{M}^{H}$.

Feeding $\mathbf{y}_{V}$ to the $V$-point DFT, $\boldsymbol{\Phi}^{H}$, yields the demodulated ZP-SEFDM signal as:

$$
\begin{aligned}
\mathbf{r}_{z p} & =\boldsymbol{\Phi}^{H} \mathbf{y}_{V}=\mathbf{M}^{H}\left(\tilde{\mathbf{H}}_{z p} \tilde{\mathbf{x}}+\mathbf{z}_{v}\right)=\mathbf{M}^{H} \mathbf{M} \mathbf{\Lambda}_{z p} \mathbf{M}^{H} \tilde{\mathbf{F}} \mathbf{s}+\boldsymbol{\Phi}^{H} \tilde{\mathbf{z}}_{z p} \\
& =\boldsymbol{\Lambda}_{z p} \boldsymbol{\Gamma} \mathbf{s}+\tilde{\mathbf{z}}_{\Phi}=\boldsymbol{\lambda}_{z p} \odot \tilde{\mathbf{s}}+\tilde{\mathbf{z}}_{\Phi}
\end{aligned}
$$

where $\boldsymbol{\lambda}_{z p}$ is the subchannel gains vector and $\tilde{\mathbf{z}}_{\Phi}$ is the $V \times 1$ AWGN noise vector. Equation (28), analogous to (12) for OFDM systems, provides evidence that using ZP instead of a CP in SEFDM allows the decomposition of the wideband multipath channel into independent narrowband channels.

\section{A. Channel Estimation and Equalization in ZP-SEFDM}

The analytical expression of estimated channel characteristics for ZP-SEFDM are given by:

$$
\begin{aligned}
\hat{\boldsymbol{\lambda}}_{z p} & =\mathbf{r}_{z p \cdot / \tilde{\mathbf{s}}} \\
& =\left(\boldsymbol{\lambda}_{z p} \odot \tilde{\mathbf{s}}+\tilde{\mathbf{z}}_{\Phi}\right) \cdot / \tilde{\mathbf{s}}=\underbrace{\lambda_{z p}}_{1}+\underbrace{\tilde{\mathbf{z}}_{\Phi} \cdot / \tilde{\mathbf{s}}}_{2}
\end{aligned}
$$

where part 1 , describes the channel gains while part 2 corresponds to the noise vector. Equation (29) shows that there is no self-created ICI enhancement between the subcarriers. The analytical expression of the equalization process is given as:

$$
\hat{\mathbf{r}}_{z p}=\mathbf{r}_{z p} \cdot / \boldsymbol{\lambda}_{z p}=\underbrace{\tilde{\mathbf{s}}}_{1}+\underbrace{\tilde{\mathbf{z}}_{\Phi} \cdot / \boldsymbol{\lambda}_{z p}}_{2}
$$

Unlike the case of CP-SEFDM equation of (21), equation (30) demonstrates that equalizing the channel effects in ZPSEFDM signals does not lead to additional interference between the subchannels.

\section{Performance Results}

Simulations are carried out based on the narrowband internetof-things (NB-IoT) standard [12] appropriately modified to accommodate the bandwidth compression factor $(\alpha)$ [16], [17]. The system parameters are: $N=12$ data subcarriers, $Q=128$ [16] and the modulation format is quadrature phase shift keying.

The frame structure is similar to those of LTE [12] where every radio frame of length $10 \mathrm{~ms}$ consists of ten equally sized subframes, each of which contains $2 \times 0.5 \mathrm{~ms}$ time slots. In every time slot, there are seven SEFDM symbols. Of these seven symbols, one carries a pilot (the first symbol in the time slot) that is used for channel estimation and the other six symbols carry data signals. The pilot symbols are the demodulation reference symbol (DMRS) [12].

The MSE in channel estimation, error vector magnitude (EVM) and bit error rate (BER) are computed for both $\mathrm{CP}$ SEFDM and ZP-SEFDM systems using 5G-new radio (5GNR) tapped delayed line (TDL) channel model of type (D). The taps delays and power values are given in the Table 7.7.24 of the standards in [18] and the channel model parameters is following the work in [19]. The MSE is given by [10]:

$$
\operatorname{MSE}=\mathbb{E}\left\{[\boldsymbol{\lambda}-\hat{\boldsymbol{\lambda}}]^{H}[\boldsymbol{\lambda}-\hat{\boldsymbol{\lambda}}]\right\}
$$

where $\boldsymbol{\lambda}$ is the gain of the channel under evaluation and $\hat{\lambda}$ is the estimated channel gain at the receiver. From figure 1, it is evident that the MSE of the channel estimation in CPSEFDM eventually reaches a non-zero error floor for any value of $\alpha<1$, as corroborated in (20). On the contrary, the MSE for the ZP method monotonically decreases with improved $E_{b} / N_{o}$, which agrees with our mathematical findings in (29).

The EVM is defined as [20]:

$$
\mathrm{EVM} \hat{=} \sqrt{\frac{\mathbb{E}\left\{|\hat{\mathbf{r}}-\mathbf{r}|^{2}\right\}}{N . \mathbb{E}\left\{|\mathbf{r}|^{2}\right\}}}
$$

where $\mathbf{r}$ is an SEFDM symbol, $\hat{\mathbf{r}}$ is the SEFDM symbol after traversing a noisy multipath channel, and $N$ is the number 


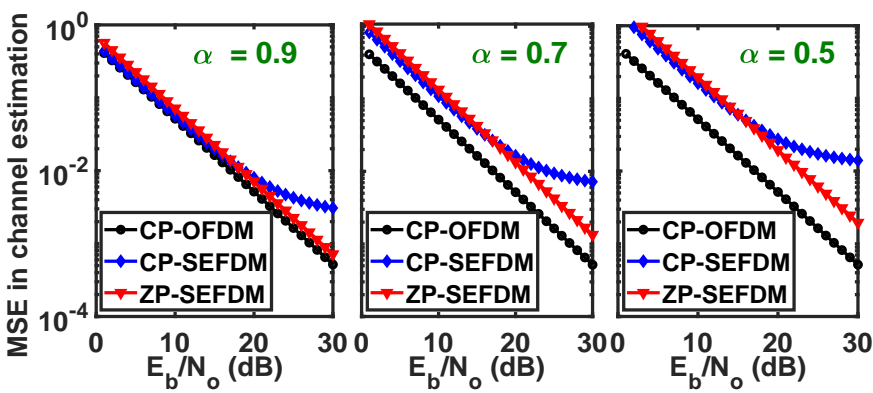

Fig. 1. MSE results using TDL-D channel model.

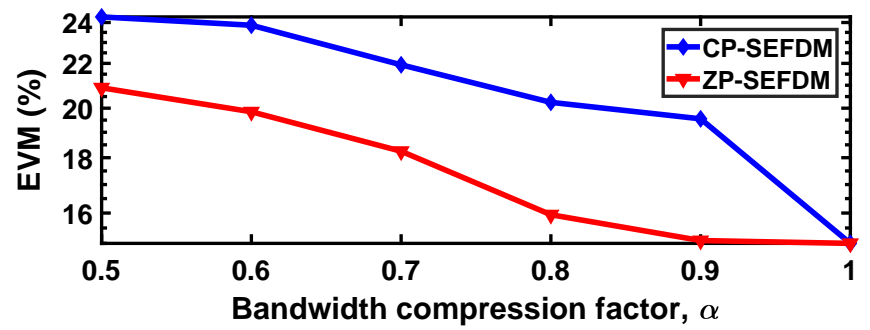

Fig. 2. EVM results using TDL-D channel model.

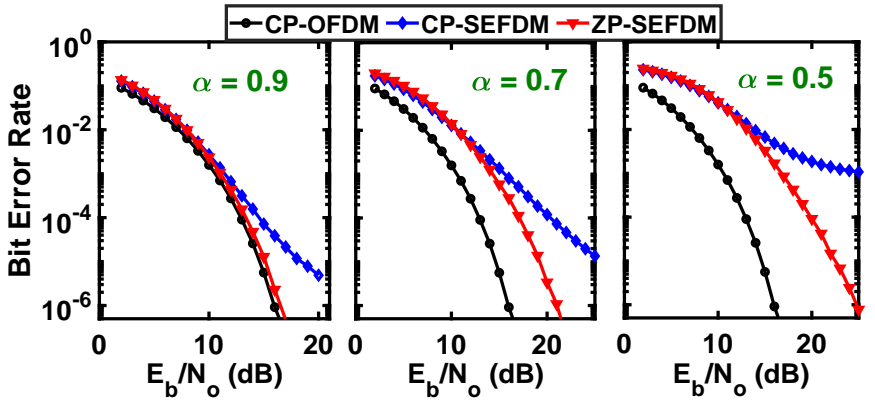

Fig. 3. BER results using TDL-D channel model.

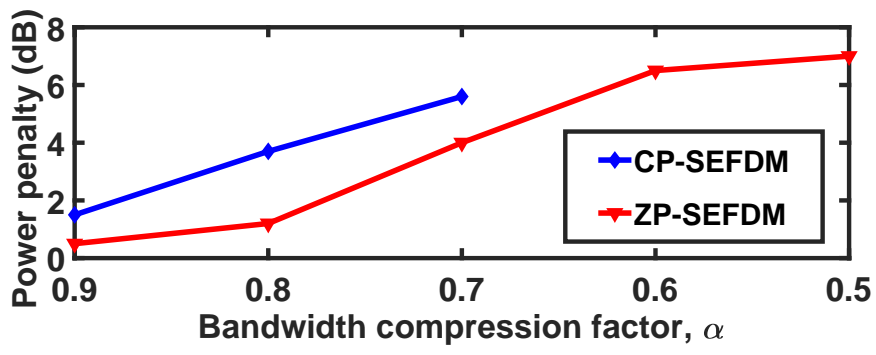

Fig. 4. $E_{b} / N_{o}$ difference between SEFDM and OFDM systems.

of active subcarriers. EVM is the chosen figure-of-merit to evaluate the distortion in the complex received signal that affects signal reliability. In Fig. 2, EVM performances are depicted at $E_{b} / N_{o}=15 d B$. From the EVM plots, it is clear that ZP-SEFDM outperforms CP-SEFDM for various values of $\alpha$ factor. ZP-SEFDM has lower EVM values because the narrowband subchannels, at each subcarrier, are orthogonal to each other. This matches the analytical findings in (21) and (30) for CP-SEFDM and ZP-SEFDM, respectively.

In addition BER is measured for OFDM and SEFDM systems with different $\alpha$ values. In this work, a sphere decoder [6] is used to decode the equalized complex symbols. Fig. 3 shows that, unlike ZP-SEFDM, the CP-SEFDM BER curves diverge and reach a non-zero error floor for all values of $\alpha$.

The power penalty, defined as the $E_{b} / N_{o}$ difference be- tween OFDM and SEFDM systems, is measured in $(\mathrm{dB})$ at BER $=10^{-4}$ and shown in Fig. 4. The CP-SEFDM curve stops at $\alpha=0.7$ because the BER performance reaches error rate higher than $10^{-4}$ for $\alpha<0.7$.

\section{CONCLUSION}

In addition to the ISI effects caused by multipath channels, non-orthogonal multi-carrier systems, such as SEFDM, also suffer from self-induced ICI. This work therefore proposes a novel channel estimation and equalization technique employing an orthogonal DFT-based demodulator in conjunction with ZP. Simulation results show that ZP-SEFDM can offer over one order of magnitude improvement in MSE performance at high $E_{b} / N_{o}$ values compared to CP-SEFDM with commensurate improvement in received signal EVM and BER.

\section{REFERENCES}

[1] B. Muquet, Zhengdao Wang, G. B. Giannakis, M. de Courville, and P. Duhamel, "Cyclic prefixing or zero padding for wireless multicarrier transmissions?" IEEE Trans. Commun., vol. 50, no. 12, pp. 2136-2148, Dec 2002.

[2] S. Venkatesan and R. A. Valenzuela, "OFDM for 5G: Cyclic prefix versus zero postfix, and filtering versus windowing," in Proc. IEEE Int. Conf. Commun. (ICC), May 2016, pp. 1-5.

[3] G. Wunder et al., "5GNOW: non-orthogonal, asynchronous waveforms for future mobile applications," IEEE Commun. Mag., vol. 52, no. 2, pp. 97-105, February 2014.

[4] F.-L. Luo and C. Zhang, Signal processing for 5G: algorithms and implementations. John Wiley \& Sons, 2016.

[5] M. Vaezi, Z. Ding, and H. V. Poor, Multiple Access Techniques for $5 G$ Wireless Networks and Beyond. Springer, 2019.

[6] I. Kanaras, A. Chorti, M. R. D. Rodrigues, and I. Darwazeh, "Spectrally Efficient FDM Signals: Bandwidth Gain at the Expense of Receiver Complexity," in Proc. IEEE Int. Conf. Commun., June 2009, pp. 1-6.

[7] M. Nakao and S. Sugiura, "Spectrally Efficient Frequency Division Multiplexing With Index-Modulated Non-Orthogonal Subcarriers," IEEE Wireless Commun. Lett., vol. 8, no. 1, pp. 233-236, Feb 2019.

[8] S. V. Zavjalov, S. V. Volvenko, and S. B. Makarov, "A Method for Increasing the Spectral and Energy Efficiency SEFDM Signals," IEEE Commun. Lett., vol. 20, no. 12, Dec 2016.

[9] H. Ghannam and I. Darwazeh, "Robust Channel Estimation Methods for Spectrally Efficient FDM Systems," in Proc. IEEE 87th Veh. Technol. Conf. (VTC Spring), June 2018, pp. 1-6.

[10] T. Xu and I. Darwazeh, "Transmission Experiment of Bandwidth Compressed Carrier Aggregation in a Realistic Fading Channel," IEEE Trans. Veh. Technol., vol. 66, no. 5, pp. 4087-4097, May 2017.

[11] D. Nopchinda et al., "Dual Polarization Coherent Optical Spectrally Efficient Frequency Division Multiplexing," IEEE Photon. Technol. Lett., vol. 28, no. 1, pp. 83-86, Jan 2016.

[12] 3GPP, "LTE; Evolved Universal Terrestrial Radio Access; Physical layer procedures," 3GPP, TS 36.213, Rel. 14, v.14.2.0, Apr. 2017.

[13] A. Goldsmith, Wireless Communications. Cambridge University Press, 2005.

[14] R. M. Gray et al., "Toeplitz and circulant matrices: A review," Foundations Trends ${ }^{\circledR}$ Commun. Inf. Theory, vol. 2, no. 3, pp. 155-239, 2006.

[15] Xianbin Wang, P. Ho, and Y. Wu, "Robust channel estimation and ISI cancellation for OFDM systems with suppressed features," IEEE J. Sel. Areas Commun., vol. 23, no. 5, pp. 963-972, May 2005.

[16] T. Xu, C. Masouros, and I. Darwazeh, "Waveform and Space Precoding for Next Generation Downlink Narrowband IoT," IEEE Internet Things J., vol. 6, no. 3, pp. 5097-5107, June 2019.

[17] W. Ozan et al., "Time Precoding Enabled Non-Orthogonal Frequency Division Multiplexing," in Proc. 30th IEEE Int. Symp. on Personal, Indoor and Mobile Radio Communications, Sep. 2019, pp. 1-6.

[18] 3GPP, "5G; Study on channel model for frequencies from 0.5 to 100 GHz," 3GPP, TR 38.901, Rel. 14, v.14.0.0, May 2017.

[19] H. Ghannam and I. Darwazeh, "A Proposal for Scalable 5G New Radio Frames with Enhanced Throughput," in Proc. IEEE 89th Veh. Technol. Conf. (VTC Spring), April 2019, pp. 1-6.

[20] I. Kotzer, S. Har-Nevo, S. Sodin, and S. Litsyn, "An analytical approach to the calculation of EVM in clipped multi-carrier signals," IEEE Trans. Commun., vol. 60, no. 5, 2012. 